\title{
Some study of Thermoelastic Steady State behavior of Thick annular disc with Internal Heat Generation
}

\author{
C. M. Bhongade ${ }^{1}$ and M. H. Durge ${ }^{2}$ \\ ${ }^{I}$ Department of Mathematics, Shri. Shivaji College, Rajura, Maharashtra, India \\ ${ }^{2}$ Department of Mathematics, Anand Niketan College, Warora, Maharashtra, India
}

\begin{abstract}
The present paper deals with the determination of displacement and thermal stresses in a thick annular disc with internal heat generation. Arbitrary heat $f(r)$ is applied on the upper surface of disc whereas lower surface dissipates heat by convection and the fixed circular edge are thermally insulated. Here we compute the effects of internal heat generation of a thick annular disc in terms of stresses along radial direction. The governing heat conduction equation has been solved by the method of integral transform technique. The results are obtained in a series form in terms of Bessel's functions. The results for temperature change, displacement and stresses have been computed numerically and illustrated graphically.
\end{abstract}

Keywords Thermal stresses, internal heat generation, annular disc, steady state.

\section{Introduction}

Deshmukh [1] studied transient heat conduction problem in a thin hollow cylinder and determined thermal stresses. Gogulwar and Deshmukh [2] studied the inverse problem of thermal stresses in a thin annular disc. Kulkarni and Deshmukh [3] has determined the quasi-static steady state thermal stresses in thick annular disc. Shang sheng $\mathrm{Wu}[6]$ studied the direct thermoelastic problem in an annular fin with its base subjected to a heat flux of a decayed exponential function of time. In this paper thick annular disc is considered and discussed its thermoelasticity with the help of the Goodier's thermoelastic displacement potential function and the Michell's function. To obtain the temperature distribution integral transform method is applied. The results are obtained in series form in terms of Bessel's functions and the temperature change, displacement function and stresses have been computed numerically and illustrated graphically. Here we compute the effects of internal heat generation in terms of stresses along radial direction. A mathematical model has been constructed of a thick annular disc with the help of numerical illustration by considering copper (pure) disc. No one previously studied such type of problem. This is new contribution to the field.

The direct problem is very important in view of its relevance to various industrial mechanics subjected to heating such as the main shaft of lathe, turbines and the role of rolling mill, base of furnace of boiler of a thermal power plant, gas power plant and the measurement of aerodynamic heating.

\section{Formulation of the Problem}

Consider a thick annular disc of thickness $2 \mathrm{~h}$ defined by $a \leq r \leq b,-h \leq z \leq h$. An arbitrary heat $f(r)$ is applied on the upper surface of the disc $(z=h)$ and heat dissipates by convection from the lower boundary surface $(\mathrm{z}=-\mathrm{h})$ into the surrounding at the zero temperature. The circular edge $(r=a$ and $r=b)$ are thermally insulated. Assume that the boundary of the annular disc is free from traction. Under these prescribed conditions, the quasi-static steady state thermal stresses are required to be determined.

The differential equation governing the displacement potential function $\phi(r, z)$ is given in [4] as

$$
\frac{\partial^{2} \phi}{\partial r^{2}}+\frac{1}{r} \frac{\partial \phi}{\partial r}+\frac{\partial^{2} \phi}{\partial z^{2}}=K \tau
$$

where $\mathrm{K}$ is the restraint coefficient and temperature change $\tau=T-T_{i}, T_{i}$ is initial (ambient) temperature. Displacement function $\phi$ is known as Goodier's thermoelastic displacement potential.

The steady state temperature of the plate satisfies the heat conduction equation,

$$
\frac{\partial^{2} T}{\partial r^{2}}+\frac{1}{r} \frac{\partial T}{\partial r}+\frac{\partial^{2} T}{\partial z^{2}}+\frac{q}{k}=0
$$

with the boundary conditions

$$
\begin{aligned}
& \frac{\partial T}{\partial r}=0 \quad \text { at } r=a, \quad-h \leq z \leq h \\
& \frac{\partial T}{\partial r}=0 \quad \text { at } r=b, \quad-h \leq z \leq h \\
& \frac{\partial T}{\partial z}+h_{s_{1}} T=f(r) \quad \text { at } z=h, a \leq r \leq b \\
& \frac{\partial T}{\partial z}-h_{s_{2}} T=0 \quad \text { at } z=-h, a \leq r \leq b
\end{aligned}
$$


where $\mathrm{k}$ is the thermal conductivity of the material of the disc, $\mathrm{q}$ is internal heat generation, $h_{s_{1}}$ and $h_{s_{2}}$ are the relative heat transfer coefficients on the upper and lower surface of the thick annular disc.

The Michell's function M must satisfy

$$
\nabla^{2} \nabla^{2} M=0
$$

where

$$
\nabla^{2}=\frac{\partial^{2}}{\partial r^{2}}+\frac{1}{r} \frac{\partial}{\partial r}+\frac{\partial^{2}}{\partial z^{2}}
$$

The components of the stresses are represented by the thermoelastic displacement potential $\phi$ and Michell's function $\mathrm{M}$ as

$$
\begin{aligned}
& \sigma_{r r}=2 G\left\{\frac{\partial^{2} \phi}{\partial r^{2}}-K \tau+\frac{\partial}{\partial z}\left[v \nabla^{2} M-\frac{\partial^{2} M}{\partial r^{2}}\right]\right\} \\
& \sigma_{\theta \theta}=2 G\left\{\frac{1}{r} \frac{\partial \phi}{\partial r}-K \tau+\frac{\partial}{\partial z}\left[v \nabla^{2} M-\frac{1}{r} \frac{\partial M}{\partial r}\right]\right\} \\
& \sigma_{z z}=2 G\left\{\frac{\partial^{2} \phi}{\partial z^{2}}-K \tau+\frac{\partial}{\partial z}\left[(2-v) \nabla^{2} M-\frac{\partial^{2} M}{\partial z^{2}}\right]\right\}
\end{aligned}
$$

and

$$
\sigma_{r z}=2 G\left\{\frac{\partial^{2} \phi}{\partial r \partial z}+\frac{\partial}{\partial r}\left[(1-v) \nabla^{2} M-\frac{\partial^{2} M}{\partial z^{2}}\right]\right\}
$$

where $\mathrm{G}$ and $\mathrm{v}$ are the shear modulus and Poisson's ratio respectively.

The boundary conditions on the traction free surface of an annular disc are

$$
\sigma_{r r}=\sigma_{r z}=0 \text { at } r=a \text { and } z= \pm h
$$

\section{Solution}

\subsection{Temperature change}

To obtain the expression for temperature $T(r, z)$, we introduce the finite Hankel transform over the variable $\mathrm{r}$ and its inverse transform defined as in [5]

$$
\begin{aligned}
\bar{T}\left(\beta_{m}, z\right) & =\int_{r^{\prime}=a}^{b} r^{\prime} K_{0}\left(\beta_{m}, r^{\prime}\right) T\left(r^{\prime}, z\right) d r^{\prime} \\
T(r, z) & =\sum_{m=1}^{\infty} K_{0}\left(\beta_{m}, r\right) \bar{T}\left(\beta_{m}, z\right) \\
\text { where } K_{0}\left(\beta_{m}, r\right) & =\frac{R_{0}\left(\beta_{m}, r\right)}{\sqrt{N}}, \\
R_{0}\left(\beta_{m}, r\right) & =\frac{J_{0}\left(\beta_{m} r\right)}{\beta_{m} J_{0}^{\prime}\left(\beta_{m} b\right)}-\frac{Y_{0}\left(\beta_{m} r\right)}{\beta_{m} Y_{0}^{\prime}\left(\beta_{m} b\right)}
\end{aligned}
$$

The normality constant

$$
N=\frac{b^{2}}{2} R_{0}\left(\beta_{m}, b\right)^{2}-\frac{a^{2}}{2} R_{0}\left(\beta_{m}, a\right)^{2}
$$

and $\beta_{1}, \beta_{2} \ldots .$. are roots of the transcendental equation

$$
\frac{J_{1}\left(\beta_{m} a\right)}{J_{1}\left(\beta_{m} b\right)}-\frac{Y_{1}\left(\beta_{m} a\right)}{Y_{1}\left(\beta_{m} b\right)}=0
$$

where $J_{n}(x)$ is Bessel function of the first kind of order $\mathrm{n}$ and $Y_{n}(x)$ is Bessel function of the second kind of order $n$.

On applying the finite Hankel transform defined in the Eq. (14) and its inverse transform defined in (15) to the Eq. (2), one obtains the expression for temperature as

$$
\begin{aligned}
T(r, z)= & \sum_{m=1}^{\infty} \frac{1}{\sqrt{N}}\left[\frac{J_{0}\left(\beta_{m} r\right)}{\beta_{m} J_{0}{ }^{\prime}\left(\beta_{m} b\right)}-\frac{Y_{0}\left(\beta_{m} r\right)}{\beta_{m} Y_{0}\left(\beta_{m} b\right)}\right]\left\{-\frac{1}{\left(\beta_{m}^{2}+h_{s_{1}} h_{s_{2}}\right) \sinh \left(2 \beta_{m} \mathrm{~h}\right)+\beta_{m}\left(h_{s_{1}}+h_{s_{2}}\right) \cosh \left(2 \beta_{m} \mathrm{~h}\right)}\right. \\
& \times\left[\begin{array}{l}
\left(\frac{\mathrm{dA}\left(\beta_{m}, \mathrm{~h}\right)}{\mathrm{dz}}+h_{s_{1}} \mathrm{~A}\left(\beta_{m}, \mathrm{~h}\right)-\mathrm{F}\left(\beta_{m}\right)\right)\left\langle\beta_{m} \cosh \left[\beta_{m}(z+\mathrm{h})\right]+h_{s_{2}} \sinh \left[\beta_{m}(z+\mathrm{h})\right]\right\rangle \\
+\left(\frac{\mathrm{dA}\left(\beta_{m},-\mathrm{h}\right)}{\mathrm{d} \mathrm{z}}-h_{s_{2}} \mathrm{~A}\left(\beta_{m},-\mathrm{h}\right)\right)\left\langle-\beta_{m} \cosh \left[\beta_{m}(z-\mathrm{h})\right]+h_{s_{1}} \sinh \left[\beta_{m}(z-\mathrm{h})\right]\right\rangle
\end{array}\right] \\
& \left.+\mathrm{A}\left(\beta_{m}, \mathrm{z}\right)\right\}
\end{aligned}
$$

$A\left(\beta_{m}, z\right)$ is particular integral of differential equation (2) and $\mathrm{F}\left(\beta_{m}\right)$ is the Hankel transform of $\mathrm{f}(\mathrm{r})$.

$F\left(\beta_{m}\right)=\int_{r^{\prime}=a}^{b} \frac{r^{\prime}}{\sqrt{N}}\left[\frac{J_{0}\left(\beta_{m} r^{\prime}\right)}{\beta_{m} J_{0}\left(\beta_{m} b\right)}-\frac{Y_{0}\left(\beta_{m} r^{\prime}\right)}{\beta_{m} Y_{0}\left(\beta_{m} b\right)}\right] f\left(r^{\prime}\right) d r^{\prime}$

Michells function M

Now suitable form of $\mathrm{M}$ which satisfy Eq. (7) is given by

$$
\begin{aligned}
& M=K \sum_{m=1}^{\infty} \frac{F\left(\beta_{m}\right)}{\sqrt{N}}\left[\frac{J_{0}\left(\beta_{m} r\right)}{\beta_{m} J_{0}\left(\beta_{m} b\right)}-\frac{Y_{0}\left(\beta_{m} r\right)}{\beta_{m} Y_{0}\left(\beta_{m} b\right)}\right] \\
& \times\left\{B_{m}\left(\begin{array}{c}
\beta_{m} \cosh \left[\beta_{m}(z+\mathrm{h})\right] \\
+h_{s_{2}} \sinh \left[\beta_{m}(z+\mathrm{h})\right]-\beta_{m} \mathrm{e}^{\beta_{m}(z+\mathrm{h})}
\end{array}\right)\right. \\
& \left.+C_{m} \beta_{m}(z+\mathrm{h})\left(\begin{array}{c}
-\beta_{m} \cosh \left[\beta_{m}(z-\mathrm{h})\right]+h_{s_{1}} \sinh \left[\beta_{m}(z-\mathrm{h})\right] \\
+\cosh \left(2 \beta_{m} \mathrm{~h}\right) \beta_{m} \mathrm{e}^{\beta_{m}(z+\mathrm{h})}+h_{s_{1}} \sinh \left(2 \beta_{m} \mathrm{~h}\right) \mathrm{e}^{\beta_{m}(z+\mathrm{h})}
\end{array}\right)\right\}
\end{aligned}
$$

where $B_{m}$ and $C_{m}$ are arbitrary functions. 
3.2 Goodiers thermoelastic displacement potential $\phi$ Assuming the displacement function $\phi(r, z)$ which satisfies Eq. (1) as

$$
\begin{aligned}
\phi(r, z)= & \sum_{m=1}^{\infty} \frac{K}{\sqrt{N\left[\left(\beta_{m}^{2}+h_{s_{1}} h_{s_{2}}\right) \sinh \left(2 \beta_{m} \mathrm{~h}\right)+\beta_{m}\left(h_{s_{1}}+h_{s_{2}}\right) \cosh \left(2 \beta_{m} \mathrm{~h}\right)\right]}}\left[\frac{J_{0}\left(\beta_{m} r\right)}{\beta_{m} J_{0}\left(\beta_{m} b\right)}-\frac{Y_{0}\left(\beta_{m} r\right)}{\beta_{m} Y_{0}\left(\beta_{m} b\right)}\right] \\
& \times\left\{-\left(\frac{\mathrm{dA}\left(\beta_{m}, \mathrm{~h}\right)}{\mathrm{d} z}+h_{s_{1}} \mathrm{~A}\left(\beta_{m}, \mathrm{~h}\right)-\mathrm{F}\left(\beta_{m}\right)\right)\right. \\
& \times\left[\begin{array}{c}
\left.\beta_{m} \cosh \left[\beta_{m}(z+\mathrm{h})\right]+h_{s_{2}} \sinh \left[\beta_{m}(z+\mathrm{h})\right]\right]-\left(\frac{\mathrm{dA}\left(\beta_{m},-\mathrm{h}\right)}{\mathrm{dz}}-h_{s_{2}} \mathrm{~A}\left(\beta_{m},-\mathrm{h}\right)\right) \\
-\beta_{m} \mathrm{e}^{\beta_{m}(z+\mathrm{h})}
\end{array}\right. \\
& \times\left[\begin{array}{c}
-\beta_{m} \cosh \left[\beta_{m}(z-\mathrm{h})\right]+h_{s_{1}} \sinh \left[\beta_{m}(z-\mathrm{h})\right] \\
\left.\left.+\beta_{m} \cosh \left(2 \beta_{m} \mathrm{~h}\right) \mathrm{e}^{\beta_{m}(z+\mathrm{h})}+h_{s_{1}} \sinh \left(2 \beta_{m} \mathrm{~h}\right) \mathrm{e}^{\beta_{m}(z+\mathrm{h})}\right]\right\}
\end{array}\right.
\end{aligned}
$$

Now using Eqs. (20), (22) and (23) in Eq. (9), (10), (11) \& (12), one obtains the expressions for stresses respectively as

We set for convenience

$$
\begin{aligned}
& g_{1}(r)=\frac{J_{1}{ }^{\prime}\left(\beta_{m} r\right)}{\beta_{m} J_{0}{ }^{\prime}\left(\beta_{m} b\right)}-\frac{Y_{1}^{\prime}\left(\beta_{m} r\right)}{\beta_{m} Y_{0}^{\prime}\left(\beta_{m} b\right)}, \\
& g_{2}(r)=\frac{J_{0}\left(\beta_{m} r\right)}{\beta_{m} J_{0}{ }^{\prime}\left(\beta_{m} b\right)}-\frac{Y_{0}\left(\beta_{m} r\right)}{\beta_{m} Y_{0}{ }^{\prime}\left(\beta_{m} b\right)}, \\
& g_{3}(r)=\frac{J_{0}^{\prime}\left(\beta_{m} r\right)}{\beta_{m} J_{0}^{\prime}\left(\beta_{m} b\right)}-\frac{Y_{0}^{\prime}\left(\beta_{m} r\right)}{\beta_{m} Y_{0}{ }^{\prime}\left(\beta_{m} b\right)} \text {, } \\
& U=\left[\left(\beta_{m}{ }^{2}+h_{s_{1}} h_{s_{2}}\right) \sinh \left(2 \beta_{m} \mathrm{~h}\right)+\beta_{m}\left(h_{s_{1}}+h_{s_{2}}\right) \cosh \left(2 \beta_{m} \mathrm{~h}\right)\right], \\
& N=\frac{\mathrm{dA}\left(\beta_{m}, \mathrm{~h}\right)}{\mathrm{dz}}+h_{s_{1}} \mathrm{~A}\left(\beta_{m}, \mathrm{~h}\right)-\mathrm{F}\left(\beta_{m}\right) \text {, } \\
& \mathrm{L}=\frac{\mathrm{dA}\left(\beta_{m},-\mathrm{h}\right)}{\mathrm{dz}}-h_{s_{2}} \mathrm{~A}\left(\beta_{m},-\mathrm{h}\right) \text {, } \\
& u_{1}=\left[J_{0}\left(\beta_{m} a\right) Y_{0}{ }^{\prime}\left(\beta_{m} b\right)-Y_{0}{ }^{\prime}\left(\beta_{m} a\right) J_{0}{ }^{\prime}\left(\beta_{m} b\right)\right] \text {, } \\
& v_{1}=\left[J_{1}^{\prime}\left(\beta_{m} a\right) Y_{0}{ }^{\prime}\left(\beta_{m} b\right)-Y_{1}^{\prime}\left(\beta_{m} a\right) J_{0}{ }^{\prime}\left(\beta_{m} b\right)\right] \text {. } \\
& \frac{\sigma_{r r}}{K}=2 G \sum_{m=1}^{\infty}\left\{\frac { g _ { 1 } ( r ) } { U \sqrt { N } } \left[N\left[\beta_{m} \cosh \left[\beta_{m}(z+\mathrm{h})\right]+h_{s_{2}} \sinh \left[\beta_{m}(z+\mathrm{h})\right]-\beta_{m} \mathrm{e}^{\beta_{m}(z+\mathrm{h})}\right]\right.\right. \\
& +L\left[\begin{array}{c}
-\beta_{m} \cosh \left(\beta_{m}(z-\mathrm{h})\right]+h_{s_{1}} \sinh \left[\beta_{m}(z-\mathrm{h})\right] \\
+\beta_{m} \cosh \left(2 \beta_{m} \mathrm{~h}\right) \mathrm{e}^{\beta_{m}(z+\mathrm{h})}+h_{s_{1}} \sinh \left(2 \beta_{m} \mathrm{~h}\right) \mathrm{e}^{\beta_{m}(z+\mathrm{h})}
\end{array}\right]
\end{aligned}
$$

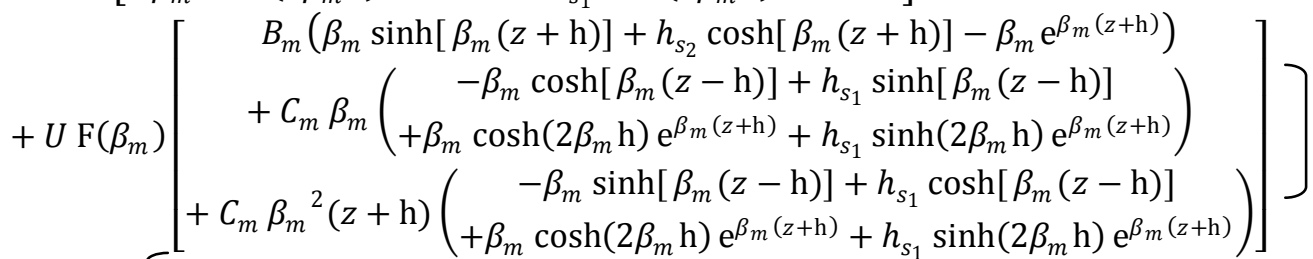

$$
\begin{aligned}
& +\frac{g_{2}(r)}{U \sqrt{N}}\left[N\left[\beta_{m} \cosh \left[\beta_{m}(z+\mathrm{h})\right]+h_{s_{2}} \sinh \left[\beta_{m}(z+\mathrm{h})\right]-\beta_{m} \mathrm{e}^{\beta_{m}(z+\mathrm{h})}\right]\right. \\
& +L\left[-\beta_{m} \cosh \left[\beta_{m}(z-\mathrm{h})\right]+h_{s 1} \sinh \left[\beta_{m}(z-\mathrm{h})\right]\right]+\mathrm{A}\left(\beta_{m}, z\right) \\
& \left.\left.+2 v U C_{m} \beta_{m}{ }^{3} \mathrm{~F}\left(\beta_{m}\right)\left[\begin{array}{c}
-\beta_{m} \cosh \left[\beta_{m}(z-\mathrm{h})\right]+h_{s_{1}} \sinh \left[\beta_{m}(z-\mathrm{h})\right] \\
+\beta_{m} \cosh \left(2 \beta_{m} \mathrm{~h}\right) \mathrm{e}^{\beta_{m}(z+\mathrm{h})}+h_{s_{1}} \sinh \left(2 \beta_{m} \mathrm{~h}\right) \mathrm{e}^{\beta_{m}(z+\mathrm{h})}
\end{array}\right]\right]\right\} \\
& \frac{\sigma_{\theta \theta}}{K}=2 G \sum_{m=1}^{\infty}\left\{\begin{array}{l}
\frac{g_{3}(r)}{r U \sqrt{N}} \\
-\beta_{m} \cosh \left[\beta_{m}(z-\mathrm{h})\right]+h_{s} \sinh \left[\beta_{m}(z-\mathrm{h})\right]
\end{array}\right. \\
& -L\left[\begin{array}{c}
-\beta_{m} \cosh \left[\beta_{m}(z-\mathrm{h})\right]+h_{s_{1}} \sinh \left[\beta_{m}(z-\mathrm{h})\right] \\
+\beta_{m} \cosh \left(2 \beta_{m} \mathrm{~h}\right) \mathrm{e}^{\beta_{m}(z+\mathrm{h})}+h_{s_{1}} \sinh \left(2 \beta_{m} \mathrm{~h}\right) \mathrm{e}^{\beta_{m}(z+\mathrm{h})}
\end{array}\right]
\end{aligned}
$$

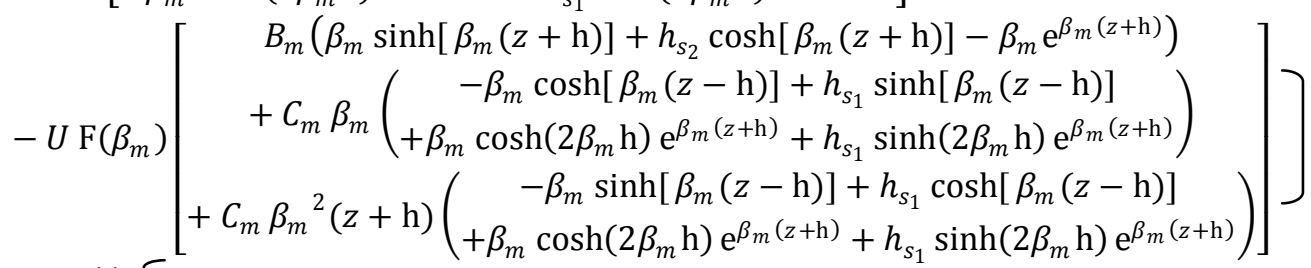

$$
\begin{aligned}
& +\frac{g_{2}(r)}{U \sqrt{N}}\left[N\left[\beta_{m} \cosh \left[\beta_{m}(z+\mathrm{h})\right]+h_{s_{2}} \sinh \left[\beta_{m}(z+\mathrm{h})\right]\right]\right. \\
& \left.\left.\begin{array}{l}
+L\left[-\beta_{m} \cosh \left[\beta_{m}(z-\mathrm{h})\right]+h_{s_{1}} \sinh \left[\beta_{m}(z-\mathrm{h})\right]\right]+\mathrm{A}\left(\beta_{m}, z\right) \\
+2 v U C_{m} \beta_{m}{ }^{3} \mathrm{~F}\left(\beta_{m}\right)\left[\begin{array}{c}
-\beta_{m} \cosh \left[\beta_{m}(z-\mathrm{h})\right]+h_{s_{1}} \sinh \left[\beta_{m}(z-\mathrm{h})\right] \\
+\beta_{m} \cosh \left(2 \beta_{m} \mathrm{~h}\right) \mathrm{e}^{\beta_{m}(z+\mathrm{h})}+h_{s_{1}} \sinh \left(2 \beta_{m} \mathrm{~h}\right) \mathrm{e}^{\beta_{m}(z+\mathrm{h})}
\end{array}\right]
\end{array}\right]\right\}
\end{aligned}
$$




$$
\begin{aligned}
& \frac{\sigma_{Z Z}}{K}=2 G \sum_{m=1}^{\infty} \frac{g_{2}(r)}{U \sqrt{N}}\left\{-N \beta_{m}{ }^{2}\left[\beta_{m} \cosh \left[\beta_{m}(z+h)\right]+h_{s_{2}} \sinh \left[\beta_{m}(z+h)\right]-\beta_{m} e^{\beta_{m}(z+h)}\right]\right. \\
& -L \beta_{m}^{2}\left[\begin{array}{c}
-\beta_{m} \cosh \left[\beta_{m}(z-\mathrm{h})\right]+h_{s_{1}} \sinh \left[\beta_{m}(z-\mathrm{h})\right] \\
+\beta_{m} \cosh \left(2 \beta_{m} \mathrm{~h}\right) \mathrm{e}^{\beta_{m}(z+\mathrm{h})}+h_{s_{1}} \sinh \left(2 \beta_{m} h\right) e^{\beta_{m}(z+h)}
\end{array}\right] \\
& +N\left[\beta_{m} \cosh \left[\beta_{m}(z+h)\right]+h_{s_{2}} \sinh \left[\beta_{m}(z+h)\right]\right] \\
& +L\left[-\beta_{m} \cosh \left[\beta_{m}(z-h)\right]+h_{s_{1}} \sinh \left[\beta_{m}(z-h)\right]\right]+\mathrm{A}\left(\beta_{m}, z\right) \\
& +U \mathrm{~F}\left(\beta_{m}\right)\left[\begin{array}{c}
(1-v) 2 C_{m} \beta_{m}{ }^{3}\left(\begin{array}{c}
-\beta_{m} \cosh \left[\beta_{m}(z-\mathrm{h})\right]+h_{s_{1}} \sinh \left[\beta_{m}(z-\mathrm{h})\right] \\
\left.+\beta_{m} \cosh \left(2 \beta_{m} \mathrm{~h}\right) \mathrm{e}^{\beta_{m}(z+\mathrm{h})}+h_{s_{1}} \sinh \left(2 \beta_{m} \mathrm{~h}\right) \mathrm{e}^{\beta_{m}(z+\mathrm{h})}\right) \\
-B_{m} \beta_{m}{ }^{3}\left(\beta_{m} \sinh \left[\beta_{m}(z+\mathrm{h})\right]+h_{s_{2}} \cosh \left[\beta_{m}(z+\mathrm{h})\right]-\beta_{m} \mathrm{e}^{\beta_{m}(z+\mathrm{h})}\right) \\
+C_{m} \beta_{m}{ }^{4}(z+\mathrm{h})\left(\begin{array}{c}
-\beta_{m} \sinh \left[\beta_{m}(z-\mathrm{h})\right]+h_{s_{1}} \cosh \left[\beta_{m}(z-\mathrm{h})\right] \\
+\beta_{m} \cosh \left(2 \beta_{m} \mathrm{~h}\right) \mathrm{e}^{\beta_{m}(z+\mathrm{h})}+h_{s_{1}} \sinh \left(2 \beta_{m} \mathrm{~h}\right) \mathrm{e}^{\beta_{m}(z+\mathrm{h})}
\end{array}\right)
\end{array}\right)
\end{array}\right\} \\
& \frac{\sigma_{r z}}{K}=2 G \sum_{m=1}^{\infty} \frac{g_{3}(r)}{U \sqrt{N}}\left\{-N \beta_{m}\left[\beta_{m} \sinh \left[\beta_{m}(z+\mathrm{h})\right]+h_{s_{2}} \cosh \left[\beta_{m}(z+\mathrm{h})\right]-\beta_{m} \mathrm{e}^{\beta_{m}(z+\mathrm{h})}\right]\right. \\
& -\beta_{m} L\left[\begin{array}{c}
-\beta_{m} \sinh \left[\beta_{m}(z-\mathrm{h})\right]+h_{s_{1}} \cosh \left[\beta_{m}(z-\mathrm{h})\right] \\
+\beta_{m} \cosh \left(2 \beta_{m} \mathrm{~h}\right) \mathrm{e}^{\beta_{m}(z+\mathrm{h})}+h_{s_{1}} \sinh \left(2 \beta_{m} \mathrm{~h}\right) \mathrm{e}^{\beta_{m}(z+\mathrm{h})}
\end{array}\right] \\
& \left.+U \beta_{m}{ }^{2} \mathrm{~F}\left(\beta_{m}\right)\left[\begin{array}{c}
-2 v C_{m}\left(\begin{array}{c}
-\beta_{m} \sinh \left[\beta_{m}(z-\mathrm{h})\right]+h_{s_{1}} \cosh \left[\beta_{m}(z-\mathrm{h})\right] \\
+\beta_{m} \cosh \left(2 \beta_{m} \mathrm{~h}\right) \mathrm{e}^{\beta_{m}(z+\mathrm{h})}+h_{s_{1}} \sinh \left(2 \beta_{m} \mathrm{~h}\right) \mathrm{e}^{\beta_{m}(z+\mathrm{h})}
\end{array}\right) \\
-C_{m}(z+\mathrm{h})\left(\begin{array}{c}
-\beta_{m} \cosh \left[\beta_{m}(z-\mathrm{h})\right]+h_{s_{1}} \sinh \left[\beta_{m}(z-\mathrm{h})\right] \\
+\beta_{m} \cosh \left(2 \beta_{m} \mathrm{~h}\right) \mathrm{e}^{\beta_{m}(z+\mathrm{h})}+h_{s_{1}} \sinh \left(2 \beta_{m} \mathrm{~h}\right) \mathrm{e}^{\beta_{m}(z+\mathrm{h})}
\end{array}\right) \\
-B_{m}\left(\begin{array}{c}
-\beta_{m} \cosh \left[\beta_{m}(z-\mathrm{h})\right]+h_{s_{1}} \sinh \left[\beta_{m}(z-\mathrm{h})\right] \\
+\beta_{m} \cosh \left(2 \beta_{m} \mathrm{~h}\right) \mathrm{e}^{\beta_{m}(z+\mathrm{h})}+h_{s_{1}} \sinh \left(2 \beta_{m} \mathrm{~h}\right) \mathrm{e}^{\beta_{m}(z+\mathrm{h})}
\end{array}\right)
\end{array}\right]\right\}
\end{aligned}
$$

In order to satisfy condition (13), solving equations (24) and (27) for $B_{m}$ and $C_{m}$ one obtains

$$
\begin{aligned}
B_{m}= & \frac{u_{1}}{U \mathrm{~F}\left(\beta_{m}\right)\left(\beta_{m}-h_{S_{2}}\right) v_{1}} \\
& \times\left[N \beta_{m}+L\left(-\beta_{m} \cosh \left(2 \beta_{m} \mathrm{~h}\right)-h_{s_{1}} \sinh \left(2 \beta_{m} \mathrm{~h}\right)+\mathrm{A}\left(\beta_{m},-\mathrm{h}\right)\right)\right] \\
C_{m}= & \frac{1}{2 v \beta_{m} U \mathrm{~F}\left(\beta_{m}\right)}\left[\frac{N\left(h_{\left.s_{2}-\beta_{m}\right)}\right)}{\left(h_{s_{1}}+\beta_{m}\right)\left(\cosh \left(2 \beta_{m} \mathrm{~h}\right)+\sinh \left(2 \beta_{m} \mathrm{~h}\right)\right)}+L\right]
\end{aligned}
$$

Setting

\section{Special case and Numerical calculations}

(1) $f(r)=\delta\left(r-r_{0}\right), r_{0}=1.5$.

where $\delta(r)$ is well known dirac delta function of argument $\mathrm{r}$.

Applying finite Hankel transform as defined in eq.(14) to the eq.(30), one obtains

$$
\begin{aligned}
& F\left(\beta_{m}\right)=\frac{r_{0}}{\beta_{m}}\left[\frac{J_{0}\left(\beta_{m} r_{0}\right)}{J_{0}^{\prime}\left(\beta_{m} b\right)}-\frac{Y_{0}\left(\beta_{m} r_{0}\right)}{Y_{0}^{\prime}\left(\beta_{m} b\right)}\right] \\
& (2) q(r, z)=\delta\left(r-r_{0}\right) \delta\left(z-z_{0}\right), z_{0}=0 . \\
& \bar{q}\left(\beta_{m}, z\right)=\frac{r_{0}}{\beta_{m}} \delta\left(z-z_{0}\right)\left[\frac{J_{0}\left(\beta_{m} r_{0}\right)}{J_{0}^{\prime}\left(\beta_{m} b\right)}-\frac{Y_{0}\left(\beta_{m} r_{0}\right)}{Y_{0}^{\prime}\left(\beta_{m} b\right)}\right] \\
& a=1 m, b=2 m, h=0.25 m, h_{s_{1}}=13 \text { and } h_{s_{2}}=17 .
\end{aligned}
$$

Material Properties

The numerical calculation has been carried out for a copper (pure) circular disc with the material properties defined as,

Thermal diffusivity $\alpha=112.34 \times 10^{-6} \mathrm{~m}^{2} \mathrm{~s}^{-1}$,

Specific heat $c_{\rho}=383 \mathrm{~J} / \mathrm{Kg}$

Thermal conductivity $k=386 \mathrm{~W} / \mathrm{m} \mathrm{K}$,

Shear modulus $G=48 \mathrm{G} \mathrm{pa}$,

Poisson ratio $\vartheta=0.3$.

Roots of Transcendental Equation 
The $\beta_{1}=3.1965, \beta_{2}=6.3123, \beta_{3}=9.445, \beta_{4}=12.5812, \beta_{5}=15.7199$ are the roots of transcendental equation $\frac{J_{1}\left(\beta_{m} a\right)}{J_{1}\left(\beta_{m} b\right)}-\frac{Y_{1}\left(\beta_{m} a\right)}{Y_{1}\left(\beta_{m} b\right)}=0$. The numerical calculation and the graph has been carried out with the help of mathematical software Matlab.

\section{Discussion}

In this paper a thick annular disc is considered which is free from traction and determined the expressions for temperature, displacement and stresses due to internal heat generation within it and we compute the effects of internal heat generation in terms of stresses along radial direction. As a special case mathematical model is constructed for considering copper (pure) disc with the material properties specified above.

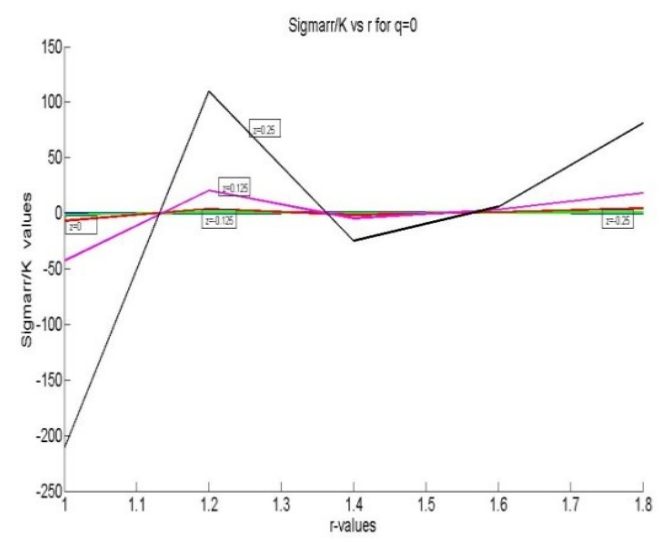

Fig. 1 Radial stress function $\frac{\sigma_{\mathrm{rr}}}{\mathrm{K}}(q=0)$.

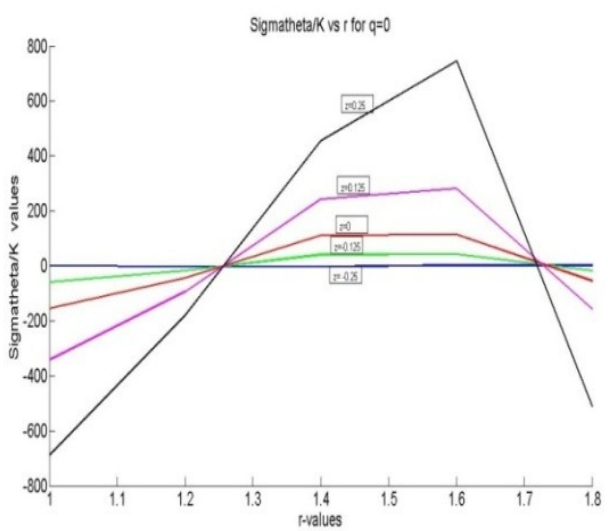

Fig. 3 Angular stress function $\frac{\sigma_{\theta \theta}}{\mathrm{K}}(q=0)$.

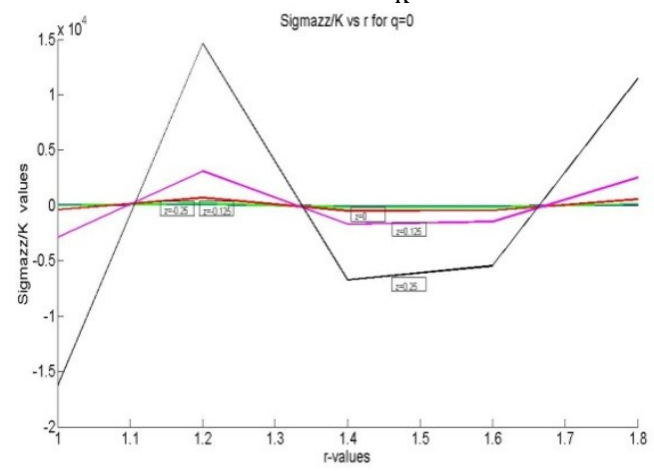

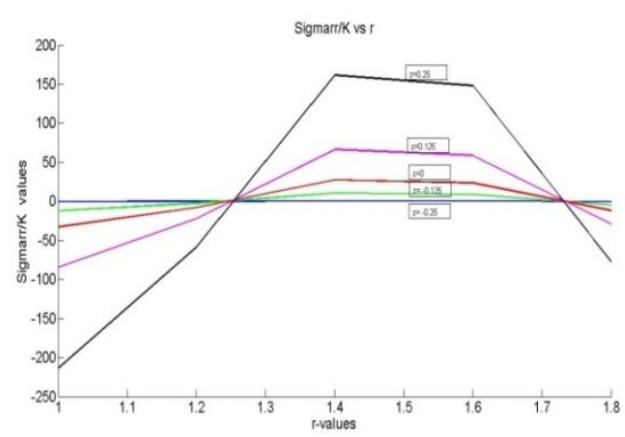

Fig. 2 Radial stress function $\frac{\sigma_{\mathrm{rr}}}{\mathrm{K}}(q \neq 0)$.

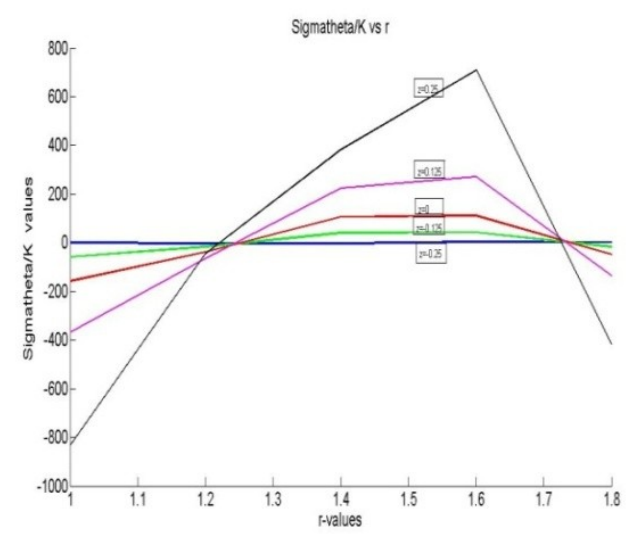

Fig. 4 Angular stress function $\frac{\sigma_{\theta \theta}}{\mathrm{K}}(q \neq 0)$.

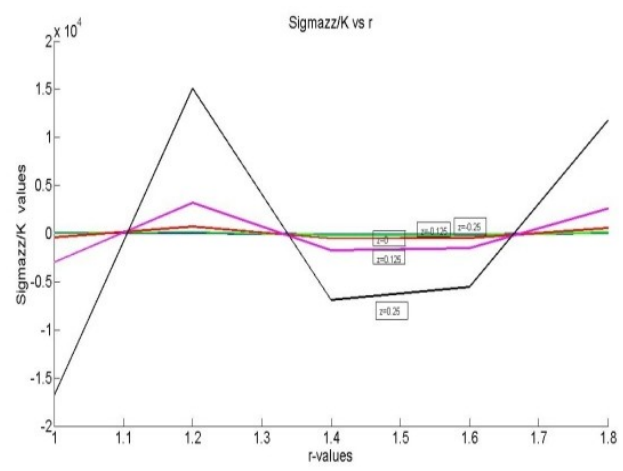


Fig. 5 Axial stress function $\frac{\sigma_{\mathrm{zz}}}{\mathrm{K}}(q=0)$.

Fig. 6 Axial stress function $\frac{\sigma_{\mathrm{zz}}}{\mathrm{K}}(q \neq 0)$.
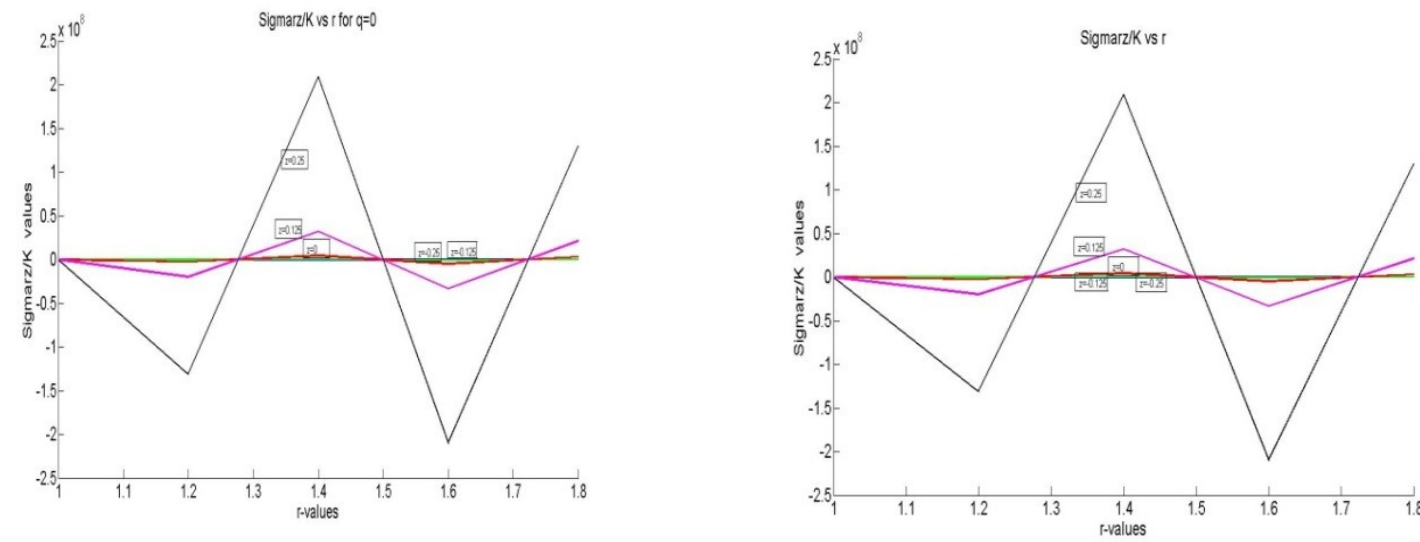

Fig. 7 Stress function $\frac{\sigma_{\mathrm{rz}}}{\mathrm{K}}(q=0)$.

Fig. 8 Stress function $\frac{\sigma_{\mathrm{rz}}}{\mathrm{K}}(q \neq 0)$.

From figure 1 and 2, it is observed that the radial stress function $\frac{\sigma_{\mathrm{rr}}}{\mathrm{K}}$ increases in radial direction due to internal heat generation in thick annular disc.

From figure 3 and 4 , it is observed that the angular stress function $\frac{\sigma_{\theta \theta}}{\mathrm{K}}$ increases slightly in radial direction due to internal heat generation in thick annular disc.

From figure 5 and 6 , it is observed that the axial stress function $\frac{\sigma_{\mathrm{zz}}}{\mathrm{K}}$ behave normally in radial direction due to internal heat generation in thick annular disc.

From figure 7 and 8 , it is observed that the stress function $\frac{\sigma_{\mathrm{rz}}}{\mathrm{K}}$ behave normally in radial direction due to internal heat generation in thick annular disc.

\section{Conclusion}

We can summarize that due to internal heat generation in thick annular disc the radial stress and the angular stress function are increases in radial direction whereas the axial stress function and the stress function behave normally.

The results obtained here are useful in engineering problems particularly in the determination of state of stress in a thick annular disc and base of furnace of boiler of a thermal power plant and gas power plant and the measurement of aerodynamic heating.

\section{References}

[1] Deshmukh K.C., " Generalized transient heat conduction problem in a thin hollow cylinder", Far East Journal of Applied Mathematics, 6(3), 253-264, (2002).

[2] Gogulwar V. S. and Deshmukh K.C., "An inverse quasi-static thermal stresses in an annular disc", Proceeding of ICADS, Narosa Publishing House, New Delhi, (2002).

[3] Kulkarni V. S. and Deshmukh K.C., "Quasi- static steady state thermal stresses in thick annular disc", Journal of thermal stresses, 31,331-342, (2008).

[4] Naotake Noda, Richard B Hetnarski and Yoshinobu Tanigawa, “ Thermal Stresses”, $2^{\text {nd }}$ edn. 259-261, Taylor, and Francis, New York, (2003).

[5] Ozisik M. N., “ Boundary value problem of heat conduction”, International Text Book Company, Scranton, Pennsylvania, 135-148, (1968).

[6] Shang-sheng Wu, “Analysis on transient thermal stresses in an annular fin”, Journal of Thermal Stresses, 20, 591-615, (1997). 